\title{
Fatores associados a sintomas depressivos em idosos atendidos em ambulatório público de Geriatria
}

\author{
Factors associated with depressive symptoms in elderly assisted in a Geriatric outpatient clinic
}

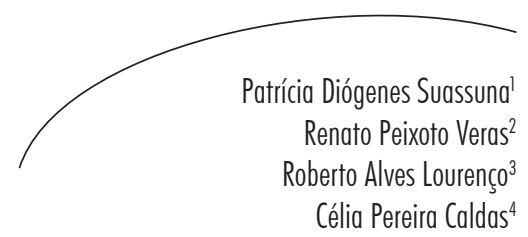

\section{Resumo}

Objetivo: Investigar fatores biopsicossociais associados à presença de sintomatologia depressiva em idosos atendidos em ambulatório público de Geriatria, na cidade do Rio de Janeiro, Brasil. Método: Estudo retrospectivo, de revisão de prontuários dos idosos atendidos na Policlínica Piquet Carneiro, no período de $1^{\circ}$ de maio de 2004 a 31 de abril de 2006. Como instrumento de pesquisa, foi elaborado um roteiro para transcrever as informações contidas nos prontuários. Os dados foram analisados utilizando-se programa SAS version 9.1, e a técnica de regressão utilizada foi a regressão logística multinomial (stepwise). Resultados: Aproximadamente 53\% da população apresentaram sintomas depressivos. $\mathrm{Na}$ análise multivariada, identificou-se associação significativa entre a sintomatologia depressiva e as variáveis: idade, distúrbio do sono, mobilidade e equilíbrio, e número de enfermidades crônicas presentes. Após o ajuste do modelo, permaneceram as variáveis idade (OR=2,8 IC 95\% 3,1 - 2,4) e mobilidade e equilíbrio $(\mathrm{OR}=1,8 \mathrm{IC}$ 95\% 2,0 - 1,52). Conclusão: A idade, o número de comorbidades e as alterações de mobilidade e equilíbrio estão associados à sintomatologia depressiva neste grupo.

\section{Abstract}

Objective: Identify bio-psychosocial factors associated with depressive symptoms in elderly attended in a public outpatients unit, in Rio de Janeiro, Brazil. Method: It is a retrospective study based on medical records. Data were analyzed using the program SAS version 9.1 and multinomial logistic regression (stepwise). Results: Approximately $53 \%$ of the population presented depressive symptoms. Multivariate analysis identified significant association between depressive symptoms and the following variables: age, sleep disorders, mobility/ balance, and quantity of chronic diseases. After fitting the

\author{
Palavras-chave: Idosos. \\ Sintomas depressivos. \\ Avaliação da Tecnologia \\ Biomédica. Ambulatório \\ hospitalar. Registros \\ médicos. Demência. Rio de
} Janeiro-Brasil.

Key words: Aged. Depressive symptoms. Biomedical Technology Assessment. Outpatient Hospital. Medical Records. Dementia. Rio de Janeiro - Brazil.

Programa de Pós-graduação em Ciências Médicas. Universidade do Estado do Rio de Janeiro. Rio de Janeiro, RJ, Brasil.

2 Departamento de Epidemiologia, Instituto de Medicina Social. Universidade do Estado do Rio de Janeiro. Rio de Janeiro, RJ, Brasil.

3 Departamento de Medicina Interna, Faculdade de Ciências Médicas. Universidade do Estado do Rio de Janeiro. Rio de Janeiro, RJ, Brasil.

4 Departamento de Saúde Pública, Faculdade de Enfermagem. Universidade do Estado do Rio de Janeiro. Rio de Janeiro, RJ, Brasil. 
model, age (OR=2.8 IC 95\% 3.1 - 2.4) and balance/ mobility (OR=1.8 IC 95\% 2.0 1.52) remained associated. Conclusion: Age, number of co-morbidities and balance / mobility are associated with depression symptoms in this group.

\section{INTRODUÇÃO}

A depressão é um dos transtornos mentais mais comuns entre indivíduos idosos. ${ }^{1} \mathrm{Na}$ população mundial, a prevalência de sintomas depressivos entre indivíduos com 65 anos ou mais de idade varia entre $10,3 \%$ e $13,5 \%{ }^{2}$ no Brasil, chega a $14,3 \%{ }^{3}$

Esta doença crônica eleva a probabilidade de desenvolver incapacidade funcional, uma vez que sua presença pode ser responsável pela perda da autonomia e pelo agravamento de enfermidades preexistentes.

A idade avançada, o sexo feminino, a presença de comorbidades, o uso de múltiplos medicamentos, o declínio cognitivo e do estado funcional e condições sociais precárias são frequentemente citadas entre os principais fatores de risco associados à depressão. ${ }^{4,5}$

Apesar de sua relevância clínica, sintomas depressivos em idosos são frequentemente não detectados, o que pode ser atribuído, em parte, à expressão clínica diferente desses sintomas em relação aos demais grupos etários. Há também uma baixa valorização dessas manifestações por parte dos profissionais de saúde generalistas, que geralmente focalizam sua atenção clínica no problema principal que motivou a busca de atendimento pelo paciente. ${ }^{6}$ Nesse sentido, a avaliação sistemática dos pacientes idosos, mediante a aplicação de escalas de avaliação de sintomas depressivos, poderia contribuir para melhorar a detecção dos casos de depressão nesse grupo etário.

Este estudo teve como objetivo investigar a associação de fatores biopsicossociais com a presença de sintomatologia depressiva em idosos atendidos em um ambulatório público de Geriatria, na cidade do Rio de Janeiro, Brasil.

\section{METODOLOGIA}

Foi realizado estudo do tipo retrospectivo. ${ }^{7,8}$ A população de estudo foram os indivíduos com 65 anos ou mais de idade que procuraram o Serviço de Geriatria da Universidade do Estado do Rio de Janeiro. A amostra foi constituída por todos os idosos atendidos no serviço no período compreendido entre os dias $1^{\circ}$ de maio de 2004 e 30 de abril de 2006. Aqueles que preencheram os critérios de inclusão no estudo tiveram seus prontuários analisados quanto às variáveis de estudo.

A variável dependente foi a sintomatologia depressiva. Ela foi avaliada por meio da Escala de Depressão Geriátrica 15 (EDG-15), cuja validade em idosos foi determinada para a população brasileira por Almeida \& Almeida ${ }^{6}$ e Paradela, Lourenço \& Veras. ${ }^{?}$

As variáveis independentes foram: capacidade funcional - Índice de $\operatorname{Kat}^{10}$ e Índice de Lawton; ${ }^{11}$ variáveis sociodemográficas - sexo, idade, escolaridade, estado civil e número de filhos; comorbidades crônicas - hipertensão arterial sistêmica, cardiopatias, enfermidades circulatórias, cérebro-vasculares, respiratórias e ósteo-articulares, câncer, doença de Parkinson, enfermidades mentais, catarata e glaucoma, perda auditiva, alterações gástricas, genito-urinárias e diabetes mellitus; velocidade de marcha aferida pelo teste Timed Get up and Go (TGUG); ${ }^{1}$ desempenho cognitivo - avaliado pelo MiniExame de Estado Mental (MEEM); ${ }^{12}$ distúrbios do sono; uso de medicações; fatores de risco sociais - morte de pessoa próxima, mudança de casa, 
separação conjugal na família, perda financeira, cuidado de familiar dependente e uso abusivo de álcool; índice de massa corporal (IMC). ${ }^{13}$

A coleta de dados foi realizada no período compreendido entre 18 de julho e 20 de agosto de 2008. Os critérios de inclusão foram: residir na cidade do Rio de Janeiro; possuir idade igual ou superior a 65 anos e ter ingressado no Serviço de Geriatria no período de $1^{\circ}$ de maio de 2004 a 30 de abril de 2006. Como critério de exclusão, consideraram-se: MEEM $\leq 10$ e ausência de avaliação do estado funcional e/ou de avaliação dos sintomas depressivos.

Foram localizadas e colhidas as informações de 512 prontuários. Dos prontuários localizados, $341(66,6 \%)$ preenchiam os critérios para participação no estudo.

Os dados foram analisados utilizando-se o programa $\mathrm{SAS}^{14}$ version 9.1. Descreveramse as frequências das variáveis e em seguida foi realizado um cruzamento entre a variável dependente e as variáveis independentes, avaliando-se a significância por meio do teste qui-quadrado. A quantificação do grau de associação foi por meio do $\mathrm{V}$ de Cramér. $\mathrm{O}$ modelo estatístico foi elaborado por meio da técnica de regressão logística multinomial (stepwise) e a escolha do melhor modelo por meio dos valores da Deviance.

Atendendo à Resolução no 196/96 do Conselho Nacional de Saúde, o projeto foi encaminhado e aprovado pelo Comitê de Ética do Hospital Universitário Pedro Ernesto (processo no $1.396-$ CEP/HUPE).

\section{RESULTADOS}

A amostra foi composta pelos 341 indivíduos que preencheram os critérios de inclusão, entre os quais $71,84 \%$ pertenciam ao sexo feminino, $37,82 \%$ eram viúvos, com escolaridade variando em sua maioria entre um e quatro anos, e apresentando de um a três filhos (47,50\%), segundo a tabela 1.

Cerca de 53\% da amostra apresentaram sintomatologia depressiva e 65,68\% apresentaram alteração cognitiva (tabela 2). 
Tabela 1 - Variáveis sociodemográficas de idosos atendidos no período de $1^{\circ}$ de maio de 2004 a 31 de abril de 2006, em ambulatório público de Geriatria, na cidade do Rio de Janeiro,RJ.

\begin{tabular}{|c|c|c|c|}
\hline Variáveis sociodemográficas & & $\mathrm{N}$ & $\%$ \\
\hline & Mas & 96 & 28,16 \\
\hline \multicolumn{4}{|l|}{ Sexo } \\
\hline & Fem & 245 & 71,84 \\
\hline \multirow[t]{2}{*}{ Total } & & 341 & 100,00 \\
\hline & Casado & 120 & 35,19 \\
\hline \multirow[t]{4}{*}{ Estado civil } & Viúvo & 129 & 37,82 \\
\hline & Separado & 22 & 6,45 \\
\hline & Solteiro & 39 & 11,45 \\
\hline & $\mathrm{NS} / \mathrm{NR} / \mathrm{NC}$ & 31 & 9,09 \\
\hline \multirow[t]{2}{*}{ Total } & & 341 & 100,00 \\
\hline & 0 anos & 56 & 16,42 \\
\hline \multirow[t]{5}{*}{ Escolaridade } & 1-4anos & 191 & 56,01 \\
\hline & 5-9anos & 61 & 17,89 \\
\hline & 10-12anos & 17 & 5,00 \\
\hline & $>13$ anos & 12 & 3,51 \\
\hline & $\mathrm{NS} / \mathrm{NR} / \mathrm{NC}$ & 4 & 1,17 \\
\hline \multirow[t]{3}{*}{ Total } & & 341 & 100,00 \\
\hline & 0 filhos & 26 & 7,62 \\
\hline & 1-3 filhos & 162 & 47,50 \\
\hline \multirow[t]{4}{*}{ Número de filhos } & 4-6 filhos & 96 & 28,18 \\
\hline & 7-9 filhos & 20 & 5,86 \\
\hline & $>10$ filhos & 4 & 1,17 \\
\hline & $\mathrm{NS} / \mathrm{NR} / \mathrm{NC}$ & 33 & 9,67 \\
\hline Total & & 341 & 100,00 \\
\hline
\end{tabular}

Tabela 2 - Código e descrição das categorias presentes na variável EDG e MEEM e valores e percentuais encontrados na amostra dos idosos atendidos no período de $1^{\circ}$ de maio de 2004 a 31 de abril de 2006, em ambulatório público de Geriatria, na cidade do Rio de Janeiro, RJ.

\begin{tabular}{llcc}
\hline \multicolumn{1}{c}{ Variável } & \multicolumn{1}{c}{ Descrição } & $\mathrm{N}$ & $(\%)$ \\
\hline \multirow{2}{*}{ EDG } & O indivíduo é considerado normal & 161 & 47,21 \\
& Presença de sintomas depressivos & 180 & 52,79 \\
\cline { 2 - 4 } & Total & 341 & 100,00 \\
\multirow{2}{*}{ MEEM } & Sim & 224 & 65,68 \\
& Não & 117 & 34,32 \\
\cline { 2 - 4 } & Total & 341 & 100,00 \\
\hline
\end{tabular}


$\mathrm{Na}$ análise multivariada, a presença de sintoma depressivo está associada a idade, número de comorbidades e velocidade de marcha. Após o ajuste do modelo, as variáveis idade ( $\mathrm{OR}=2,8 \mathrm{IC}$
$95 \% 3,1-2,4)$ e velocidade de marcha (OR=1,8 IC $95 \% \quad 2,0$ - 1,52) mantiveram associação estatisticamente significativa (tabela 3).

Tabela 3 - Associações entre as variáveis individuais e a variável EDG dos idosos atendidos no período de $1^{\circ}$ de maio de 2004 a 31 de abril de 2006, em ambulatório público de Geriatria, na cidade do Rio de Janeiro, RJ.

\begin{tabular}{lccc}
\hline \multicolumn{1}{c}{ Variável } & $\begin{array}{c}\text { Valor do V de Cramér } \\
\left(\text { significância do teste } \chi^{2}\right)\end{array}$ & Tipo de associação & $\begin{array}{c}\text { Marca de associação } \\
\text { significativa }\end{array}$ \\
\hline Idade & $0,1704(0,0197)$ & Leve & $\times$ \\
Número de problemas & $0,1586(0,0358)$ & Leve & $\times$ \\
Distúrbio do sono & $0,2252(0,0002)$ & Leve & $\times$ \\
GUG & $0,1067(0,0491)$ & Muito leve & $\times$ \\
\hline
\end{tabular}

Tabela 4 - Variáveis explicativas no ajuste do modelo logit para respostas múltiplas da variável resposta EDG dos idosos atendidos no período de $1^{\circ}$ de maio de 2004 a 31 de abril de 2006, em ambulatório público de Geriatria, na cidade do Rio de Janeiro, RJ.

\begin{tabular}{lccccc}
\hline \multirow{2}{*}{ Variável } & $\begin{array}{c}\text { Categoria da } \\
\text { variável }\end{array}$ & Estimativa & Erro padrão & $\begin{array}{c}\text { Valor do } \\
\text { qui-quadrado }\end{array}$ & p-valor \\
\hline Intercepto & 1 & 0,6859 & 0,3530 & 3,77 & 0,0520 \\
& 95 e mais & 21,5185 & 66316,44 & 0,00 & 0,9997 \\
\multirow{2}{*}{ Idade } & De 65 a 74 & $-0,8389$ & 0,4026 & 4,34 & 0,0372 \\
& De 75 a 84 & 1,2643 & 0,3964 & 10,18 & 0,0014 \\
& De 85 a 94 & 0,0000 & 0,0000 & - & - \\
\multirow{2}{*}{ GUG } & Anormal & 0,5984 & 0,2855 & 4,39 & 0,0361 \\
& Normal & 0,0000 & 0,0000 & - & - \\
\hline
\end{tabular}

\section{DISCUSSÃO}

A amostra, em sua maioria, é formada por idosos jovens, cerca de 70\% apresentam idade inferior a 80 anos. Entretanto, por se tratar de uma população atendida em um ambulatório geriátrico, apresenta maior comprometimento das condições de saúde quando comparada com populações na comunidade. Isto fica bem claro quando observamos aspectos como a presença de sintomas depressivos (52,79\%), em que se encontrou mais que o dobro da prevalência identificada nos trabalhos realizados por
$\operatorname{Veras}^{15}(25,75 \%)$ e Borjes ${ }^{16}(17,4 \%)$, ambos os estudos realizados com idosos residentes em seu ambiente domiciliar.

Quanto à presença de déficit cognitivo, encontrou-se em $65,68 \%$ da amostra, o que representa quase três vezes mais do que o encontrado por Ishizaki, ${ }^{17}$ que identificou déficit cognitivo em $21,8 \%$ dos idosos de uma cidade do norte do Japão. Outro estudo realizado em uma cidade do Sudeste do Brasil encontrou uma prevalência de $16,5 \%$ de transtornos cognitivos, ou seja, o presente estudo apresenta percentual 
quase quatro vezes maior. No entanto, quando comparamos a inquérito realizado em ambulatório de Geriatria, observamos que o valor da presente pesquisa é apenas um pouco mais elevado, aproximadamente $50 \%$ da amostra apresentavam déficit cognitivo. ${ }^{18,19}$

$\mathrm{Na}$ análise multivariada, a sintomatologia depressiva mostrou-se associada à idade, ao número de comorbidades e à velocidade de marcha, sendo que esta última apresentou associação significativa de grau muito leve com a presença de sintomatologia depressiva. A literatura aponta que essas duas variáveis, quando surgem em conjunto, contribuem de forma significativa para o surgimento de outros fenômenos, como quedas e incapacidades. ${ }^{5,20,21}$

Ao ajustar o modelo observou-se que as variáveis que permaneceram contribuindo para o aumento na proporção de pessoas com sintomas depressivos foram à idade e a velocidade da marcha. Apresentar o test Get Up and Go com um valor superior a 12 segundos aumenta em quase duas vezes as chances de desenvolver sintomas depressivos. Com relação a idade, identificou-se efeito diferenciado de acordo com a faixa etária.

A variável idade foi categorizada para ser avaliada de modo a não comprometer o resultado do teste. De acordo com os resultados apresentados na tabela 3, existe associação significativa de grau leve a um nível de aproximadamente $2 \%$ entre as variáveis idade e sintomatologia depressiva.
A depressão não está diretamente relacionada com o envelhecimento, entretanto, com o avançar da idade, surgem algumas características atípicas ou particularidades que predispõem ao desenvolvimento de sintomas depressivos com o envelhecimento. Por exemplo, os indivíduos idosos se encontram frequentemente em condições financeiras menos favorecidas após a aposentadoria, o que resulta em piores condições de moradia e declínio social; há diminuição da possibilidade de desempenhar papel produtivo, o que geralmente leva à desmoralização e perda de status; além disso, muitas vezes vivem sozinhos após a separação dos filhos e os inevitáveis lutos pela morte de cônjuges, parentes e amigos.

Este aspecto é intensificado nos idosos institucionalizados, uma vez que se encontram rodeados por pessoas estranhas, muitas vezes isolados da atualidade cultural e, evidentemente, podendo estar experimentando a incômoda sensação do abandono, dependência e inutilidade. ${ }^{22}$

\section{CONCLUSÃO}

É alta a prevalência de sintomas depressivos e déficit cognitivo na população estudada. A presença de sintoma depressivo mostrou-se associada a idade, número de comorbidades e velocidade da marcha.

A elevada prevalência de sintomas depressivos na população geriátrica remete à importância do planejamento de medidas preventivas, no sentido de garantir a autonomia e a independência desse grupo. 


\section{REFERÊNCIAS}

1. Podsiadlo D, Richardson S. The timed "Up and Go": a test of vasic functional mobilit for frail elderly person. J Am Geriatr Soc.1991; 39:142-48.

2. Snowdon J. How high is the prevalence of depression in old age. Rev Bras Psiquiatr. 2002 [acesso em 2008 Ago 15];24:S42-S47. Disponível em: http://www.scielo.br/pdf/rbp/v24s1/8856.pdf

3. Blay SL, Bickel H, Cooper, B. Mental illness in crossnational perspective. Results from a Brazilian and German community survey among the elderly. Soc Psychiatr Epidemiol. 1999 [acesso em 2008 Ago 15]; 26:245-51. Disponível em: http://www.ncbi.nlm.nih. gov/pubmed/1792554

4. Gazallifk L, Hallal PC, Lima MS. Depressão na população idosa: Os médicos estão pesquisando. Rev. Bras. Psiquiatr.2004 [acesso em 2008 Jun 10];26(3). Disponível em: http://www.scielo.br/pdf/rbp/ v26n3/a03v26n3.pdf

5. Oliveira DLC, Goretti LC, Pereira LSM. O desempenho de idosos institucionalizados com alteração cognitiva em atividades de vida diária e mobilidade: estudo piloto. Rev. bras. Fisioter. 2006 [acesso em 2008 Set 15];10 (1) Disponível: http:// www.scielo.br/pdf/rbfis/v10n1/v10n1a12.pdf

6. Almeida OP, Almeida SA. Confiabilidade da escala de depressão geriátrica (GDS): versão reduzida. Arq. Neuropsiquiatr.1999 [acesso em 2008 Set 15];56(2-B): 421-26. Disponível em: http://www.scielo.br/pdf/ anp/v57n2B/1446.pdf

7. Lakatos EM, Marconi MA. Metodologia Científica. 5. ed. São Paulo: Atlas; 2007.

8. Tomas JR, Nelson JK. Métodos de pesquisas em atividades físicas. São Paulo: Manole; 2002.

9. Padarela EM P, Lourenço RA, Veras RP. Validação da Escala de Depressão Geriátrica em ambulatório geral. Revista de saúde pública. 2005 [acesso em 2008 Mai 18];39(6):918-23. Disponível em: http://www. scielo.br/pdf/rsp/v39n6/26986.pdf

10. Katz S; Ford A B; Moskowitz R W, Jackson BA, Jafe $\mathrm{M}$ W. Studies of illness in te aged/ the index fo ADL: standardized measure of biological and psychosocial function. Jam. 1963;185(12):.94-9.
11. Lawton MP, Brody EM. Assenssment of older people: self-monitoring and instrumental activities of daily living. Geront.1969;.9:179-186.

12. Lourenço AR, Veras RP. Mini-exame do estado mental:características psicométricas e idosos ambulatoriais.Rev. Saúde Pública.2006 [acesso em 2008 Mai 18];40(4):1-8. Disponível em: http://www. scielosp.org/pdf/rsp/v40n4/23.pdf

13. WHO.Expert consultation. Appropriate body-mass index for Asia population and its implications for policy and intervention stratigeis. The Lancet. 2004;157-163

14. SAS Institute Inc. SAS/STAT Guide for Personal Computers, Version 6. Cary, IVC: SAS Institute Inc., 1987. 1028 p.

15. Veras RP. País jovem: com cabelos brancos. Rio de Janeiro: Relume Dumará, 1994.

16. Borjes LJ,Benedite TRB; Mazo GZ. Rastreamento Cognitivo e Sintomas depressivos em idosos iniciantes de programa de exercício físico. J Bras Psiquiatr. 2007 [acesso em 2008 Out 5];56(4): 273-79. Disponível em: http://www.scielo.br/pdf/jbpsiq/v56n4/a06v56n4.pdf

17. Ishizaki J, Meguro K, Ambo H, et al. A normative, community-based study of mini-mental statein elderly adults: the effect of age and educational level. J Gerontol B Psychol Sci Soc Sci.1998 [acesso em 2008 Out 5];53:359-63. Disponível em: http:// psychsoc.gerontologyjournals.org/cgi/content/ abstract/53/6/P359

18. CALDAS CP. Introdução a Gerontologia. In: VERAS,RP; LOURENÇO, R.(Org). Formação Humana em Geriatria e Gerontologia:Uma pespectiva interdisciplinar. Rio de Janeiro, RJ:UNATI/UERJ.2006 [acesso em 2008 Set 8];1821. Disponível em: http://www.nates.ufjf.br/novo/ revista/pdf/v009n2/atualizacao.pdf

19. Teixeira INDO, Neri AL. Envelhecimento bemsucedido: uma meta no curso da vida. Psicol. USP, São Paulo. 2008 [acesso em 2008 Set 15];19(1): 81-94. Disponível em : <http://pepsic.bvs-psi. org.br/scielo.php?script $=$ sci_arttext\&pid $=$ S1678 $51772008000100010 \& \operatorname{lng}=$ pt\&nrm $=$ iso $>$. ISSN 1678-5177. 
20. Santos DM, Sichieri R. índice de massa corporal e indicadores antropométricos de adiposidade em idosos.rev. Saúde Publica,2005 [acesso em 2008 Out 2];39(2):163-8. Disponível em: http://www.scielo.br/ pdf/rsp/v39n2/24037.pdf

21. Arcoverde $\mathrm{C}$ et al. Role of Physical Activity on the Maintenance of Cognition and Activities of Daily Living in Elderly with Alzheimer's Disease. Arq
Neuropsiquiatr 2008 [acesso em 2008 Jul 9];66(2B):323-327. Disponível em: http://www.scielo.br/ pdf/anp/v66n2b/v66n2ba07.pdf

22. Forlenza OV. Transtornos Depressivos na doença de Alzheimer: diagnóstico e tratamento. Rev Psi Clínica.1994 [acesso em 2008 Jul 9];21(3):87-94. Disponível em: http://www.scielo.br/pdf/rbp/ v22n2/a10v22n2.pdf 